\title{
Combination of analytical and experimental optical clearing of rodent specimen for detecting beta- carotene: phantom study
}

Shadi Masoumi

Mohammad Ali Ansari

Ezeddin Mohajerani

Elina A. Genina

Valery V. Tuchin 


\title{
Combination of analytical and experimental optical clearing of rodent specimen for detecting beta-carotene: phantom study
}

\author{
Shadi Masoumi, ${ }^{a}$ Mohammad Ali Ansari, ${ }^{a, *}$ Ezeddin Mohajerani, ${ }^{a}$ Elina A. Genina, ${ }^{b, c}$ and Valery V. Tuchin ${ }^{b, c, d}$ \\ aShahid Beheshti University, Laser and Plasma Research Institute, Tehran, Iran \\ bSaratov State University (National Research University), Research-Educational Institute of Optics and Biophotonics, Saratov, Russia \\ 'Tomsk State University (National Research University), Interdisciplinary Laboratory of Biophotonics, Tomsk, Russia \\ IInstitute of Precision Mechanics and Control RAS, Laboratory of Laser Diagnostics of Technical and Living Systems, Saratov, Russia
}

\begin{abstract}
Recently, compression optical clearing (OC) was applied to detect dermal carotenoid using reflection spectroscopy. To enhance the precision and accuracy of reflection spectroscopy to better detect the spectral absorption of beta-carotene inside biological phantom, here, we simultaneously use compression and immersion OC using dimethyl sulfoxide. In addition, we analytically extract the absorption coefficient of beta-carotene using diffuse reflectance spectroscopy (as an analytical OC). Our results show that the presented analytical OC can be applied alone as a noninvasive method to measure cutaneous chromophores at deep tissues. Finally, we also improve the ability of the analytical clearing method mediated with experimental OC. Our result demonstrates that the combination of analytical and experimental clearing methods enhance the ability of diffuse reflection spectroscopy for extracting the absorption coefficient of beta-carotene as one of the chromospheres inside biological phantom. @ 2018 Society of Photo-Optical Instrumentation Engineers (SPIE) [DOI: 10.1117/1.JBO.23.9.095002]

Keywords: optical clearing; compression-immersion; diffuse reflectance spectroscopy; analytical optical clearing; beta-carotene.

Paper 180387R received Jun. 30, 2018; accepted for publication Aug. 24, 2018; published online Sep. 13, 2018.
\end{abstract}

\section{Introduction}

Determining cutaneous carotenoid plays an important role in human health and nutrition studies. ${ }^{1-4}$ High-pressure liquid chromatography (HPLC) is the main in vitro method for determining the amount of beta-carotene. In addition to the high cost, some amount of beta-carotene will be lost after biopsy of tissue, and it will not give a precise value. ${ }^{3,5}$

Due to the limitation of HPLC, optical methods, such as Raman spectroscopy and reflection spectroscopy, have been utilized to determine the amount of beta-carotene in human and animal skin. The accuracy of these optical methods requires evaluation. In 2010, Ermakov and Gellermann ${ }^{6}$ investigated the correlation of in vivo skin tissue carotenoid obtained by resonance Raman spectroscopy with HPLC results. Darvin et al. ${ }^{7}$ measured the content of beta-carotene in the inner palm by in vivo resonance Raman spectroscopy.

The absorption spectrum of beta-carotene is masked by the blood and melanin absorption spectra. Strong scattering of skin complicates additional beta-carotene registration due to broadening and decreasing of absorption peaks. Therefore, one can apply optical clearing (OC) to enhance determination of beta-carotene spectra. Ermakov and Gellermann ${ }^{8}$ applied the compression mediated reflection spectroscopy to determine the amount of this choromophore. The mechanical compression results in a decrease in the value of the absorption coefficient of skin due to the decrease of total blood content at the location of compression..$^{9-11}$ Some additional decrease of light scattering is also expected due to a lower blood volume fraction (as red blood cells are strong scatterers) and an increase in packing

*Address all correspondence to: Mohammad Ali Ansari, E-mail: m_ansari@sbu ac.ir density of dermal collagen fibers. ${ }^{9,11}$ Therefore, the absorption spectrum of beta-carotene can be detectable. Nakhaeva et al. ${ }^{12}$ illustrated that the pressure exerted on the skin can weaken the effect of blood by reducing the depth of the dip in the green region of the spectrum that indicated the presence of blood in the tissue. Ansari et al. ${ }^{13}$ also reported that the mechanical compression altered the value of the absorption coefficient. These changes in optical properties can affect the reconstructed concentration of desired chromophore. In addition, the amount of transported blood depends on the value of mechanical pressure and the stiffness of tissue that affects the accuracy of compression-mediated reflectance spectroscopy. Therefore, it is important to verify the accuracy of compression-mediated reflection spectroscopy to determine cutaneous beta-carotene using other OC methods, such as immersion OC.

Polyatomic alcohol (glycerol, polypropylene glycol) and organic solvents [dimethyl sulfoxide (DMSO) and benzyl ether, benzyl alcohol, tetrahydrofuran] have been applied for immersion OC. ${ }^{14-19}$ Mao et al. ${ }^{20}$ illustrated that some hydroxyl-terminated organic components' agents (1-butanol, 1,4-butanediol, 1,3-propanediol, PEG200, PEG400, and glycerol) induce significant $\mathrm{OC}$ effects of porcine skin. Their results show that the OC effect of skin caused by these alcohols is related to the number of hydroxyl groups.

As Tuchin et al. proposed, the mechanism of immersion OC can be explained by dehydration of tissue constituents and the replacement of interstitial water with an agent that has a higher refractive index $(n) .{ }^{14,21}$ Based on experimental results presented by Rylander et al., ${ }^{17}$ application of glycerol causes a $60 \%$ decrease in the reduced scattering coefficient in the context of in vitro rodent skin. Zhu et al. ${ }^{22}$ illustrated that a combination of

$1083-3668 / 2018 / \$ 25.00$ (C) 2018 SPIE 
PEG-400 and thiazone (as immersion agents) can reduce the scattering coefficient (at a wavelength of $630 \mathrm{~nm}$ ) to $60 \%$ of the initial value after $40 \mathrm{~min}$ of topical treatment of this mixture on rat skin in vitro.

Beside immersion OC, an analytical method also can be applied for measurement of chromophore inside tissues. For example, Zonios and Dimou ${ }^{23}$ applied an analytical form of diffuse reflectance from semi-infinite phantom to determine the absorption coefficient of phantom containing polystyrene beads and hemoglobin from bovine blood. Välisuo et al. ${ }^{24}$ applied a linear model based on the differential Beer-Lambert law to efficiently map the concentrations of blood and melanin in the skin. They calculated an optimal linear model around an operating point, taking into account the spectra of the selected wavelengths, the concentrations of these chromophores in the selected operating point, and the available wavelengths.

In the analytical OC method, a linear summation of different absorption spectra of key tissue constituents (blood, melanin, beta-carotene, and water) is considered to model the measured diffused reflectance with optimum values of chromophore concentration. It seems that immersion and analytical OC can be applied to enhance the capability of compression-mediated reflection spectroscopy to better reconstruct the concentration of chromophores inside tissues.

Motivation for this paper is threefold: first, we investigate the ability of both compression and immersion OC, as experimental $\mathrm{OC}$, to improve the measurement of beta-carotene absorption. Second, we use an analytical OC method based on diffuse reflectance spectrum to detect the absorption spectrum of beta-carotene from experimental spectra. Finally, we show a new way to reconstruct effectively the absorption coefficient of beta-carotene obtained from diffuse reflection spectra based on both analytical and experimental clearing of samples. Hence, the originality of the current study is applying a combination of analytical and experimental OC methods to better measure the concentration of beta-carotene inside tissue phantom.

\section{Materials and Methods}

\subsection{Experiment Set Up}

As shown in Fig. 1, the experiment setup consists of a tungsten halogen lamp with 20-W power (HL2000; Ocean Optics Inc., Dunedin, Florida) and spectrometer (USB200, Ocean Optics)

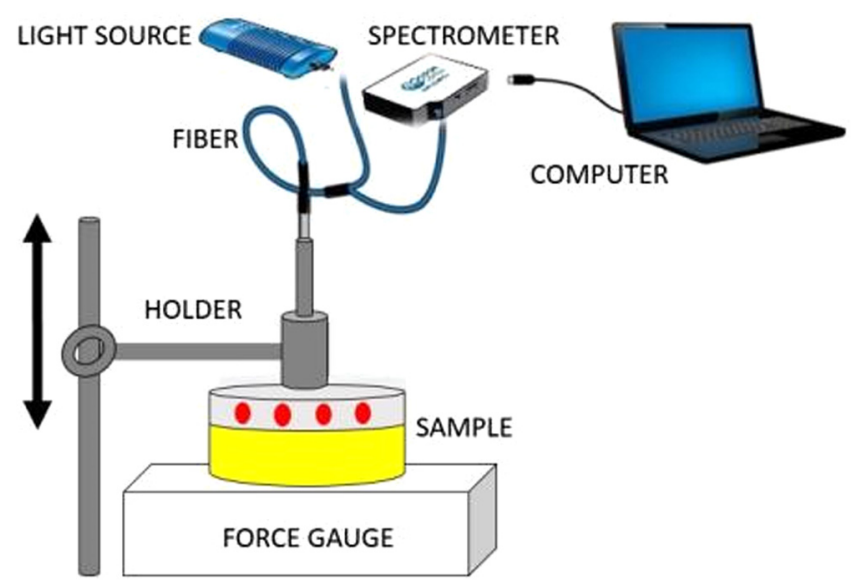

Fig. 1 The scheme of experiment setup.

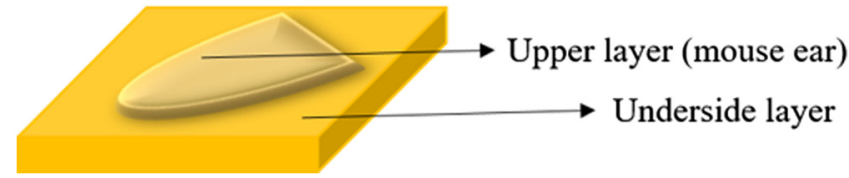

Fig. 2 A schematic of phantom. Underside layer consists of Intralipid, agar- and beta-carotene, and ear of mouse applied as an upper layer.

with a spectral range of 350 to $1100 \mathrm{~nm}$ and an optical fiber probe (R200-REF, Ocean Optics, Dunedin). This probe consists of a bundle of seven optical fibers-six illumination fibers around one read fiber-each of which is $200 \mu \mathrm{m}$ in diameter (NA $=0.22$, length $=2 \mathrm{~m}$ ) and a distance of source-detector separation of $200 \mu \mathrm{m}$. A $3^{\prime \prime} \times 0.25^{\prime \prime}$ stainless steel ferrule houses the fiber bundle. We also use a force gauge to measure the pressure.

\subsection{Phantom Preparation}

We prepared a two-layer phantom (Fig. 2). The underside layer consisted of Intralipid ${ }^{\circledR} 20 \%$ (3.3 ml), distilled water $(13 \mathrm{ml})$, agar $(0.7 \mathrm{~g})$, and beta carotene [ $\geq 97.0 \%$ (UV) Sigma-Aldrich] in different concentrations of $0.2,0.3,0.4$, and $0.6 \mu \mathrm{M}$. The upper layer is a mouse ear (2-month old, $20 \mathrm{~g}$ ) with an average thickness of $250 \mu \mathrm{m}$ (one-half of its full thickness is composed of two layers: the epidermis and the dermis, and below the skin layers, cartilage forms the structural support for the mouse ear). ${ }^{25}$ Eight ear specimens were obtained from Shahid Beheshti Medical Science University and stored at $4^{\circ} \mathrm{C}$ until experiments were performed $(15 \mathrm{~min})$. All animals participating in this experiment were cared for according to the ethic committee of Shahid Beheshti University, Iran.

\subsection{Optical Clearing Methods}

Here, we used two different OC methods to detect absorption spectra of beta-carotene from reflectance spectra. First, the method of experimental OC (compression-immersion OC) is explained, and then the method of analytical method is stated.

\subsubsection{Experimental method: compression-immersion OC}

Here, we utilized DMSO ( $\geq 99 \%$, Sigma-Aldrich) as a chemical OC agent. A solution of DMSO 50\% was prepared with ethanol as the solvent. Ear specimens were immersed in DMSO solution for $15 \mathrm{~min}$. Then, the mechanical compression was applied by fiber probe on the specimen, and the reflectance spectra were measured, as depicted in Fig. 1.

\subsubsection{Analytical method}

As mentioned in Ref. 23, the analytical form of diffuse reflectance $R(\lambda)$ from semi-infinite phantom to determine optical properties can be stated as follows:

$R(\lambda)=\frac{\mu_{s}^{\prime}(\lambda)}{k_{1}+k_{2} \mu_{a}(\lambda)}$,

where $k_{1}$ and $k_{2}$ are the parameters depended on the probe geometry and refractive indices of the studied sample and were determined to be $0.025 \mathrm{~mm}^{-1}$ and 0.075 , respectively. $\mu_{\mathrm{s}}^{\prime}(\lambda)$ is the wavelength-dependent reduced scattering coefficient, and $\mu_{\mathrm{a}}(\lambda)$ is the wavelength-dependent absorption 


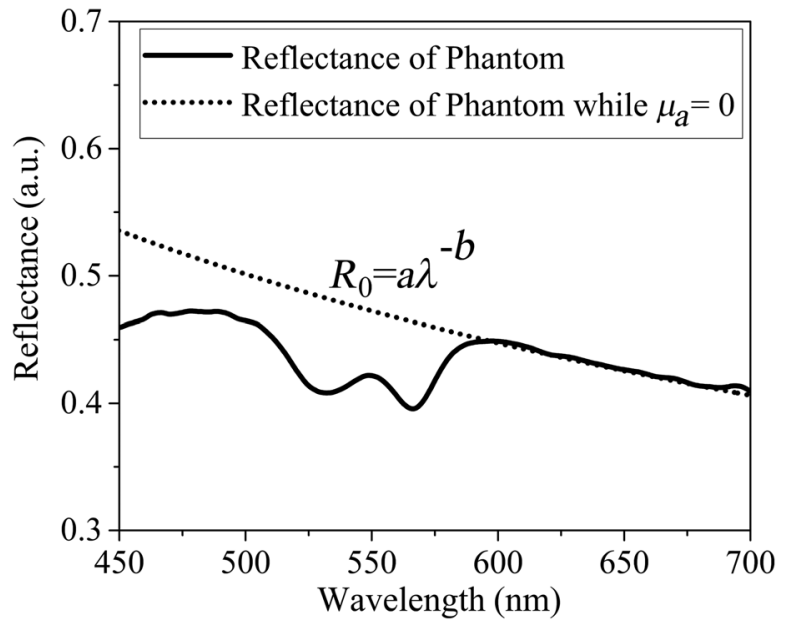

Fig. 3 Estimation of $R_{0}$ (dotted line) for measured results (solid line) using scattering power law. For the range $\lambda>600 \mathrm{~nm}$, the spectrum graph (solid line) can be fitted by the scattering power law $a \lambda^{-b}$ (dotted line), where $a=26.65$ and $b=0.63$ (with $95 \%$ confidence bounds).

coefficient of tissue-like phantom. Assume $R_{0}$ indicates the diffuse reflectance for $\mu_{\mathrm{a}}(\lambda)=0$, so $\mu_{\mathrm{s}}^{\prime}(\lambda)=k_{1} R_{0}$. This diffuse reflectance $R_{0}$ can be estimated from the wavelength region of 600 to $900 \mathrm{~nm}$ using the scattering power law, as mentioned in Ref. 26 (see Fig. 3). Finally, the absorption coefficient is obtained as follows:

$\mu_{\mathrm{a}}(\lambda)=\frac{k_{1}}{k_{2}} \cdot\left[\frac{R_{0}}{R(\lambda)}-1\right]$.

In the visible spectrum, blood and melanin are the main chromophores that affect the absorption coefficient of our phantom, while variation of water and lipid does not have significant effects in this spectrum interval. Hence, the absorption coefficient of phantom can be expressed as follows: ${ }^{27}$

$\mu_{\mathrm{a}}(\lambda)_{\text {phantom }}=M \mu_{\mathrm{a}}(\lambda)_{\text {mel }}+B \mu_{\mathrm{a}}(\lambda)_{\text {Beta }}+B l \mu_{\mathrm{a}}(\lambda)_{\text {blood }}$,

where $\mu_{\mathrm{a}}(\lambda)_{\mathrm{blood}}=\alpha B l \mu_{\mathrm{a}}(\lambda)_{\mathrm{HBO} 2}+(1-\alpha) B l \mu_{\mathrm{a}}(\lambda)_{\mathrm{HB}}$,

where $M, B$, and $B l$ denote melanin, beta-carotene, and blood volume fractions, respectively, and $\alpha$ is the degree of oxygenation of hemoglobin. The absorption spectrum of the mentioned chromophores is selected from the results presented by the "natural phenomena simulation group in University of Waterloo" and data presented by Jacques in 2013. ${ }^{28,29}$

\section{Results}

\subsection{Experimental Clearing}

Figures 4(a) and 4(b) demonstrate the effects of experimental clearing methods on diffuse reflectance and absorbance [the absorbance $A(\lambda)$ is calculated by $A(\lambda)=-\log R(\lambda)$ ]. First, we apply compression OC and study the variation of diffuse reflectance (absorbance). The results including error bars show that the removal of blood dip between 500 and $570 \mathrm{~nm}$ depends on the amount of mechanical compression. This is because the transport of blood is a function of the amount of compression and Young's module of tissue. One can see in Fig. 4(a) that, after the application of pressure and when blood is removed, the reflection increases due to less blood absorption. Indeed, scattering is also changed a bit in this range (scattering by $\mathrm{RBC}$ ), but, in this wavelength range, blood absorption prevails.

Then, we simultaneously studied the influence of both compression and a chemical clearing agent on tissue phantoms. Blue lines including error bars in Figs. 4(a) and 4(b) reveal a noticeable change in absorbance due to utilizing DMSO, as the chemical OC agent, in OC of a mouse ear. Immersion of ears in DMSO leads to a decrease in optical scattering of them, so one can see an increase in optical depth (diffusion of DMSO in phantom causes a reduction in the scattering coefficient by refractive index matching). ${ }^{30}$ Results depict that the combined OC (before $600 \mathrm{~nm}$ ) can enhance the absorption spectra, whereas these two methods have similar effects in an optical window (a wavelength longer than $600 \mathrm{~nm}$ ).

As shown in Fig. 4 and during application of OC, beta-carotene absorption spectrum appears in its specified wavelength.
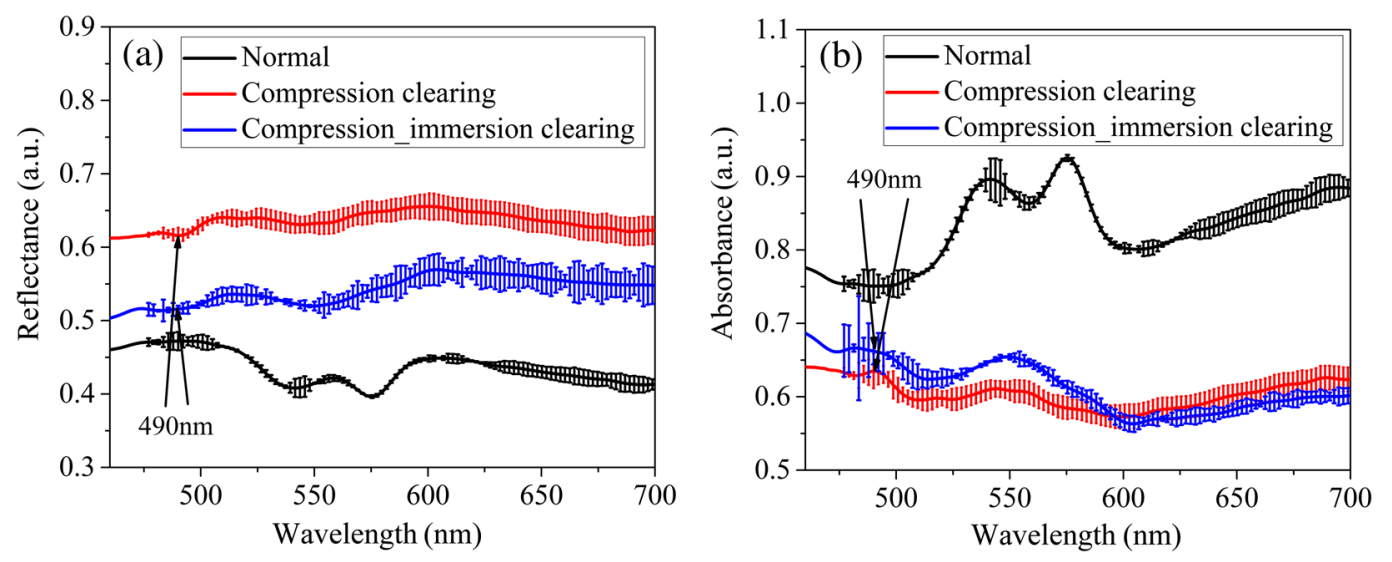

Fig. 4 The influence of compression and immersion $\mathrm{OC}$ on the spectrum of reflectance and absorbance. The black line shows the (a) reflectance and (b) absorbance before applying experimental clearing. The red line depicts the variations of reflectance (absorption) due to compression $\mathrm{OC}\left(300 \mathrm{~N} / \mathrm{m}^{2}\right)$. The blue line presents the effects of the combination of immersion and compression OC on (a) reflectance and (b) absorbance. Eight ears are used while doing the experiment for investigating the repeatability of this method. Arrows point out absorption peak of beta-carotene. 

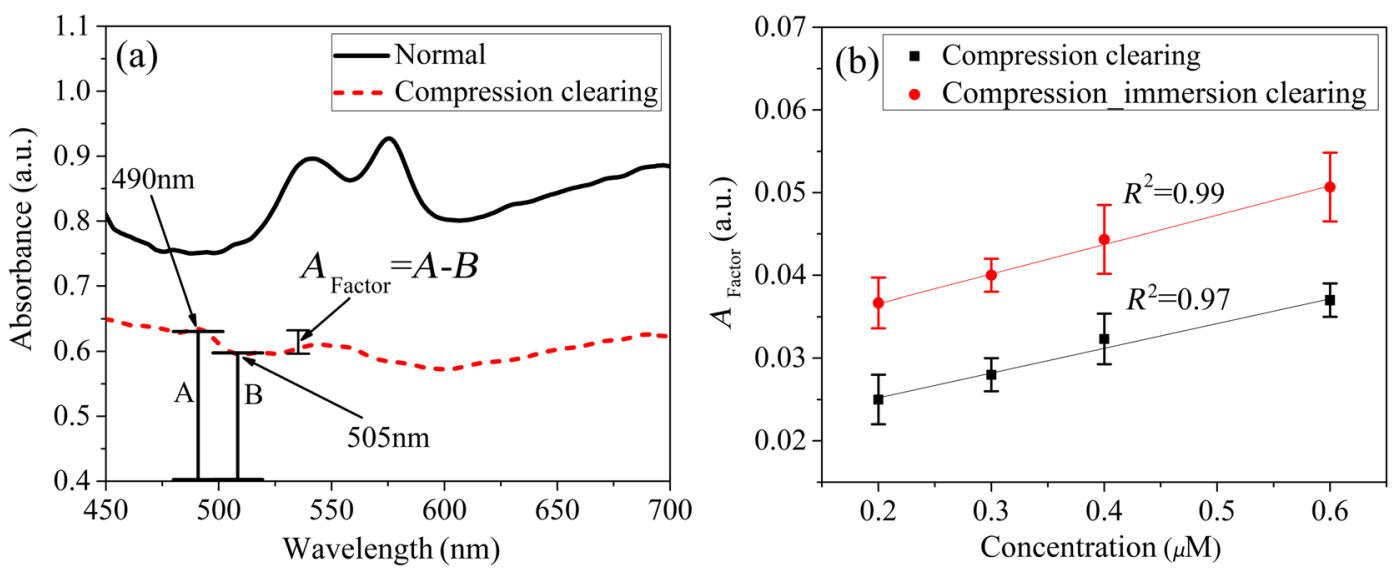

Fig. 5 (a) Illustration of $A_{\text {Factor }}$ obtained from absorbance spectra of optically cleared phantoms, where $A$ is the absorbance at the wavelength $490 \mathrm{~nm}$ and $B$ is the absorbance at the wavelength $505 \mathrm{~nm}$. The black solid line depicts the spectrum before applying compression OC, and the red dotted line illustrates the absorbance spectra of optically cleared phantom after applying compression. (b) Relation between $A_{\text {Factor }}$ and concentration of beta-carotene for different phantoms. The black line shows the influence of compression OC, and the red line presents the effects of the combination of compression and immersion OC on $A_{\text {Factor }}$.

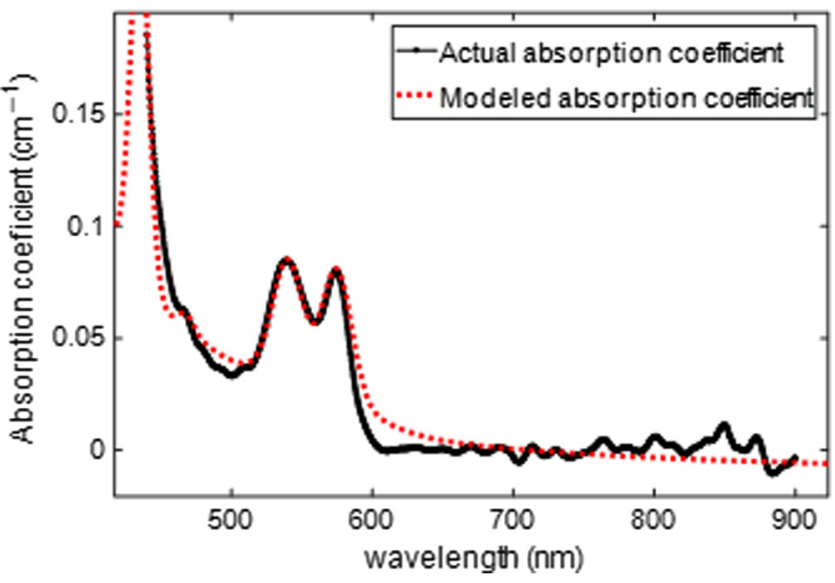

Fig. 6 Modeling measured absorption coefficient (black solid line) using diffuse reflectance presented by the red dotted line.

We define an indicator $A_{\text {Factor }}=A-B$, as shown in Fig. 5(a), to clarify the effect of OC on absorbance spectrum $(A$ is the absorbance at $\lambda=490 \mathrm{~nm}$ and $B$ is the absorbance at $\lambda=$ $505 \mathrm{~nm}$ ). Figure 5(b) depicts the amount of $A_{\text {Factor }}$ for different values of beta-carotene concentration. The results show a linear relation between the concentration of beta-carotene and $A_{\text {Factor }}$.

\subsection{Analytical Clearing}

Figure 6 compares the modeled absorption coefficient spectra and actual absorption coefficient obtained from diffuse reflectance spectra, as explained in Sec. 2.3.2. As shown in Fig. 6, these two curves are correlated with a high correlation coefficient of $R^{2}=0.94$, so the fitting parameters indicating the volume fraction of chromophores can be obtained from modeled spectra.

As shown in Fig. 7, analytical OC could extract and quantify the absorption coefficient of each chromophore within tissuelike optical phantom without experimental clearing methods for detection of beta-carotene.

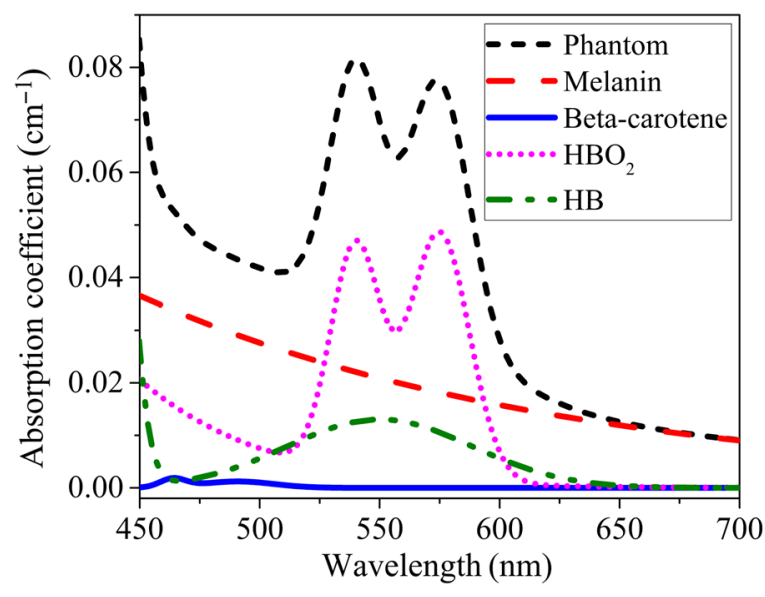

Fig. 7 Extracted absorption coefficient based on the optimum volume fraction of main chromophores.

\section{Discussion}

Here, two methods for clearing of tissue-like phantom to extract the absorption of beta-carotene are studied.

\subsection{Experimental Clearing (Compression-Immersion $O C)$}

First, we used mechanical compression to weaken the effect of high blood absorption and obtain the amount of beta-carotene in our phantoms. Ermakov and Gellermann ${ }^{8}$ investigated the correlation between results obtained by compression mediated reflection spectroscopy and Raman spectroscopy. They illustrated the feasibility of this OC to extract the absorbance spectrum of beta-carotene. Because the blood distribution inside biological tissue depends on the amount of mechanical pressure and the stiffness of tissue, the accuracy of compression mediated reflection spectroscopy can be a key limitation for detecting chromophore at deep depths.

Hence, we used DMSO as a chemical clearing agent during compression-immersion clearing to obtain beta-carotene 
absorption because DMSO is a well-known skin penetration enhancer and its permeability rate $\left(176 \pm 42 \mathrm{~g} / \mathrm{m}^{2} \mathrm{~h}\right)$ is $\sim 10$ times greater than that of water. ${ }^{17,31}$ In addition, DMSO induces a reversible conformational change of protein when it substitutes for water. ${ }^{17}$ By contrast, for example, glycerol does not have a high enough penetration rate since it can hardly penetrate into dermis to achieve OC by topical application because of the barrier of stratum corneum. ${ }^{19}$ As one can see in Fig. 4(b), applying DMSO as the OC agent improves the detection of the absorption spectrum of beta-carotene.

In this experiment, we use a phantom consisting of a mouse ear and beta-carotene at a depth of $\sim 0.2 \mathrm{~mm}$ from the surface of phantom. (In fact, we apply a bilirubin-free phantom having a low amount of melanin that influences the results.) Using DMSO and compression helps to decrease the influence of blood and mismatched refractive index to better detect betacarotene absorption spectra. The efficacy of our method to measure the beta-carotene content in human skin depends on the scattering coefficient of skin. The light scattering in skin depends on different elements, such as melanin and other tissue structures. The presence of melanin $(n=1.7)$ on the background of low refractive index of cell cytoplasm and interstitial fluid $(n=1.34)$ causes high scattering in the epidermis layer of the skin. ${ }^{32}$ However, very white skin having a low melanin content has a high scattering coefficient. ${ }^{33-35}$ Hence, in the range of 400 to $700 \mathrm{~nm}$, high absorption and scattering limits the ability of experimental and analytical OC to efficiently detect the desired chromophores.

\subsection{Analytical Clearing (Extracting the Absorption Coefficient of Beta-Carotene)}

Johansson $^{36}$ measured the reflectance spectra of a thin cerebral phantom to estimate its absorption coefficient. Using linear regression, the absorption spectra were fitted to extract the main chromophore spectrum inside cerebral phantom. We applied this idea to perform analytical OC. That means, the blood and melanin spectra were digitally removed from the measured absorption spectra, then the absorption spectra of the desired chromophore should be apparent. The spectral elimination of dominant chromophores enhanced the capability of this method to detect the low value of concentration of chromophore

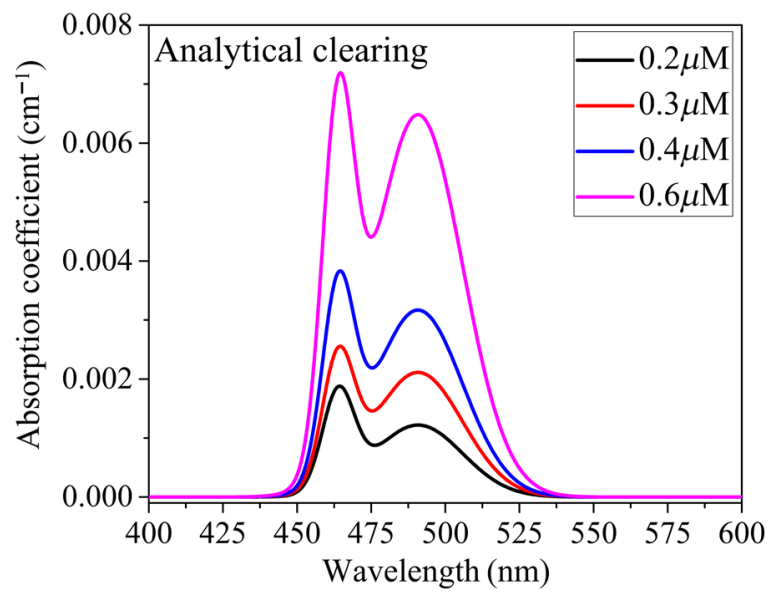

Fig. 8 Extracted spectral absorption coefficient of different values of concentration of beta-carotene using analytical OC.

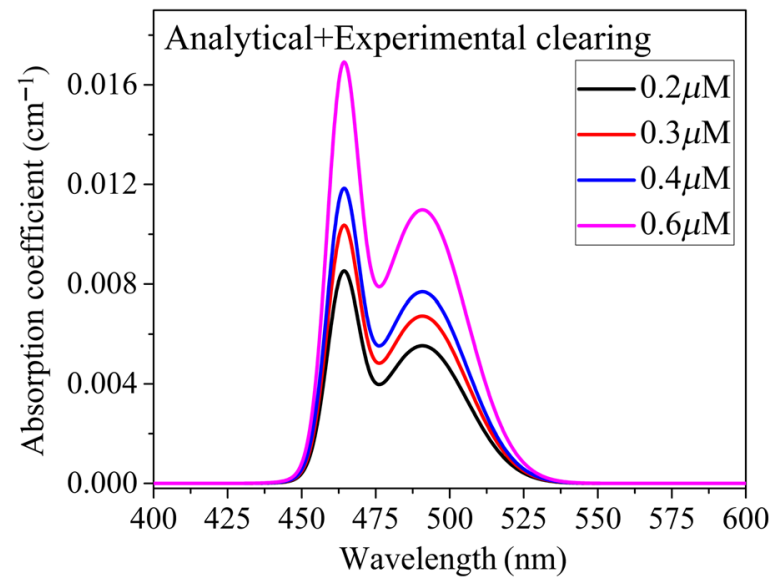

Fig. 9 Extracted spectral absorption coefficient of different values of concentration of beta-carotene using combination of analytical and experimental OC.

at deep tissues without applying any experimental clearing. For example, Fig. 8 shows the spectral absorption coefficient for different values of concentration of beta-carotene inside the phantom. In general, a combination of all three considered modalities should be helpful for improving capabilities of the OC approach because of the reduction of tissue scattering and blood absorption impact on analytical reconstruction of chromophore concentration with the masked spectrum. To prove this claim, we also combine analytical clearing with experimental clearing methods. As shown in Fig. 9, the value of the extracted absorption coefficient of beta carotene is improved. Figure 10 also compares the value of the extracted absorption coefficient at a wavelength of $490 \mathrm{~nm}$ for these two methods. It is clearly specified that the estimated value of the absorption coefficient using this hybrid method (the combination of analytical and experimental clearing) is larger than experimental or analytical clearing alone. However, compression and immersion OC should be applied when analytical $\mathrm{OC}$ is not enough to get reliable data.

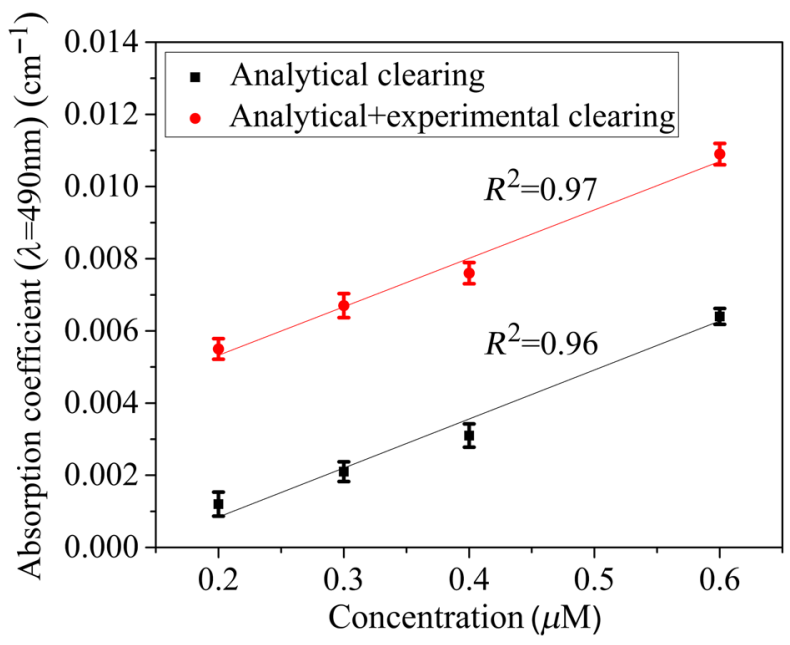

Fig. 10 Relation between extracted absorption coefficient of betacarotene at a wavelength of $490 \mathrm{~nm}$ with a concentration of betacarotene inside phantoms for analytical clearing method and combination of analytical and experimental clearing method. 


\section{Conclusion}

This study aimed to improve compression OC using the analytical OC to measure beta-carotene inside the phantom. First, we utilize compression-immersion OC to decrease both the scattering coefficient of specimen and the effect of blood spectra. Our results show that this method enhances the ability of reflectance spectroscopy to predict the amount of beta-carotene. While the accuracy of this method depends on the value of pressure and the stiffness of the biological tissue, we also apply analytical OC to detect beta-carotene. Results depict that the analytical OC can measure the absorption coefficient of beta-carotene. Indeed, the analytical OC does not require any chemical agent or compression that might change or destroy the structure of tissues (as a noninvasive method). We finally apply the experimental clearing method besides analytical clearing to improve analytical capabilities of the OC approach for the extraction absorption coefficient of beta-carotene. The results show that analytical OC can be applied independently and in combination with compression or immersion, or both, when it is needed. The presented OC techniques can be applied for determining chromophores, such as melanin and lipid, in deep tissues that have good abilities for in vivo oncology and nutrition research, especially for spectral imaging of tumors.

\section{Disclosures}

The authors have no relevant financial interests in this article and no potential conflicts of interest to disclose.

\section{Acknowledgments}

E. A. G. and V. V. T. was supported by RFBR under Grant No. 17-02-00358 and the RF Governmental under Grant No. 14.Z50.31.0044.

\section{References}

1. M. C. Meinke et al., "Comparison of different cutaneous carotenoid sensors and influence of age, skin type, and kinetic changes subsequent to intake of a vegetable extract," J. Biomed. Opt. 21(10), 107002 (2016).

2. M. E. Darvin et al., "Optical methods for noninvasive determination of carotenoids in human and animal skin," J. Biomed. Opt. 18(6), 061230 (2013).

3. A. J. Speek, C. R. Temalilwa, and J. Schrijver, "Determination of $\beta$-carotene content and vitamin A activity of vegetables by high-performance liquid chromatography and spectrophotometry," Food Chem. 19(1), 65-74 (1986).

4. J. T. Kumpulainen and J. T. Salonen, Natural Antioxidants and Anticarcinogens in Nutrition, Health and Disease, RSC, Cambridge (1999).

5. L. A. Chandler and S. J. Schwartz, "HPLC separation of cis- trans carotene isomers in fresh and processed fruits and vegetables," J. Food Sci. 52(3), 669-672 (1987).

6. I. V. Ermakov and W. Gellermann, "Validation model for Raman based skin carotenoid detection," Arch. Biochem. Biophys. 504(1), 40-49 (2010).

7. E. M. Darvin et al., "Comparison of two methods for noninvasive determination of carotenoids in human and animal skin: Raman spectroscopy versus reflection spectroscopy," J. Biophotonics 5(7), 550-558 (2012).

8. I. V. Ermakov and W. Gellermann, "Dermal carotenoid measurements via pressure mediated reflection spectroscopy," J. Biophotonics 5(7), 559-570 (2012).

9. E. A. Genina et al., "Optical clearing of biological tissues: prospects of application in medical diagnostics and phototherapy," J. Biomed. Photonics Eng. 1(1), 22-58 (2015).

10. C. R. Idelson et al., "Effect of mechanical optical clearing on nearinfrared spectroscopy," Lasers Surg. Med. 47(6), 495-502 (2015).
11. V. V. Tuchin, Optical Clearing of Tissues and Blood, SPIE Press, Bellingham, Washington (2006).

12. I. A. Nakhaeva et al., "The effect of external mechanical compression on in vivo water content in human skin," Opt. Spectrosc. 118(5) 834-840 (2015).

13. M. A. Ansari et al., "Study of the effect of mechanical pressure on determination of position and size of tumor in biological phantoms," Appl. Opt. 52(12), 2739-2749 (2013).

14. V. V. Tuchin et al., "Light propagation in tissues with controlled optical properties," J. Biomed. Opt. 2(4), 401-418 (1997).

15. L. Chen et al., "An effective balanced optical clearing method for intact biomedical imaging," Sci. Rep. 7(1), 12218 (2017).

16. J. Seo et al., "Clearing and labeling techniques for large-scale biological tissues," Mol. Cells 39 (6), 439-446 (2016).

17. C. G. Rylander et al., "Dehydration mechanism of optical clearing in tissue," J. Biomed. Opt. 11(4), 041117 (2006).

18. T. Yu et al., "Quantitative analysis of dehydration in porcine skin for assessing mechanism of optical clearing," J. Biomed. Opt. 16(9), 095002 (2011).

19. X. Wen et al., "In vivo skin optical clearing by glycerol solutions: mechanism," J. Biophotonics 3(1-2), 44-52 (2010).

20. Z. Mao et al., "Influence of alcohols on the optical clearing effect of skin in vitro," J. Biomed. Opt. 13(2), 021104 (2008).

21. D. Zhu et al., "Recent progress in tissue optical clearing," Laser Photonics Rev. 7(5), 732-757 (2013).

22. D. Zhu et al., "Imaging dermal blood flow through the intact rat skin with an optical clearing method," J. Biomed. Opt. 15(2), 026008 (2010).

23. G. Zonios and A. Dimou, "Modeling diffuse reflectance from semiinfinite turbid media: application to the study of skin optical properties," Opt. Exp. 14(19), 8661-8674 (2006).

24. P. Välisuo et al., "New closed-form approximation for skin chromophore mapping," J. Biomed. Opt. 16(4), 046012 (2011).

25. P. So et al., "Two-photon deep tissue ex vivo imaging of mouse dermal and subcutaneous structures," Opt. Express 3(9), 339-350 (1998).

26. S. Andree, C. Reble, and J. Helfmann, "Spectral in vivo signature of carotenoids in visible light diffuse reflectance from skin in comparison to ex vivo absorption spectra," Photonics Lasers Med. 2(4), 323-335 (2013).

27. W. F. Cheong, S. A. Prahl, and A. J. Welch, "A review of the optical properties of biological tissues," IEEE. J. Quantum. Electron. 26(12) 2166-2185 (1990).

28. http://www.npsg.uwaterloo.ca/index.php.

29. S. L. Jacques, "Optical properties of biological tissues: a review," Phys. Med. Biol. 58(11), R37 (2013).

30. A. K. Bui et al., "Revisiting optical clearing with dimethyl sulfoxide (DMSO)," Lasers Surg. Med. 41(2), 142-148 (2009).

31. C. Ursin et al., "Permeability of commercial solvents through living human skin," Am. Ind. Hyg. Assoc. J. 56(7), 651-660 (1995).

32. N. Sudheendran et al., "Assessment of tissue optical clearing as a function of glucose concentration using optical coherence tomography," J. Innov. Opt. Health Sci. 3(03), 169-176 (2010).

33. D. Yudovsky and L. Pilon, "Retrieving skin properties from in vivo spectral reflectance measurements," J. Biophotonics 4(5), 305-314 (2011).

34. G. Zonios et al., "Skin melanin, hemoglobin, and light scattering properties can be quantitatively assessed in vivo using diffuse reflectance spectroscopy," J. Invest. Dermatol. 117(6), 1452-1457 (2001).

35. R. R. Anderson and J. A. Parrish, "The optics of human skin," J. Invest. Dermatol. 77(1), 13-19 (1981).

36. J. D. Johansson, "Spectroscopic method for determination of the absorption coefficient in brain tissue," J. Biomed. Opt. 15(5), 057005 (2010).

Shadi Masoumi received her BS degree in atomic physics from Semnan University and her MS degree in photonics from Shahid Beheshti University, in 2018. She wrote her master thesis on tissue optical clearing methods in combination with diffuse reflection spectroscopy. Currently, she is a research assistant at laser and plasma research institute at Shahid Beheshti University, Iran. Her research interests include biomedical optics and biophotonics.

Mohammad Ali Ansari is assistant professor of photonics at Laser and Plasma Research Institute of Shahid Beheshti University, and 
head of Optical Bioimaging Lab. He obtained his PhD in photonics from Shahid Beheshti Univertity, Tehran, Iran, at 2010. His research areas includes fNIRS, diffuse optical tomography and photoacoustic imaging.

Ezeddin Mohajerani is professor of photonics at Laser and Plasma Research Institute of Shahid Beheshti University, and head of POMP Lab. He obtained his PhD in physics from University of Reading at UK in 1992. His research interest includes biophotonics, biospectroscopy, organic nanophotonics, organic nonlinear optics, smart organic materials, integrated optics, and organic optoelectronics. One of the active fields in his lab is studying noninvasive optical diagnosis and therapy of skin diseases and agriculture products based on spectroscopy. He has authored more than 150 articles, more than 10 in the field of tissue optics, optical diagnosis and therapy in Persian and English.

Elina A. Genina is a professor in the Departments of Optics and Biophotonics at Saratov State University. She is a coauthor of more than 250 peer-reviewed publications, nine book chapters, and nine patents. Her research interests include biomedical optics, laser medicine, and development of methods for control of tissue optical properties. She is a scientific secretary of International Symposium on Optics and Biophotonics (Saratov Fall Meeting). She has 2302 citations and h-index 22 (WoS, September 4, 2018).

Valery V. Tuchin is a professor and head of optics and biophotonics at Saratov State University (National Research University of Russia) and several other universities. His research interests include tissue optics, laser medicine, tissue optical clearing, and nanobiophotonics. $\mathrm{He}$ is a fellow of SPIE and OSA, has been awarded Honored Science Worker of the Russia, honored professor of Saratov University, SPIE educator award, FiDiPro (Finland), Chime Bell Prize of Hube Province (China), and Joseph W. Goodman Book Writing Award (OSA/SPIE). He has 20607 citations and h-index 66 (Google Scholar, September 4, 2018). 